\title{
Detection of nanoparticles in carbon arc discharge with laser-induced incandescence
}

\author{
S. Yatom ${ }^{1, *}$, J. Bak $^{2}$, A. Khrabryi ${ }^{1}$, Y. Raitses ${ }^{1}$ \\ ${ }^{1}$ Princeton Plasma Physics Laboratory, Princeton, NJ 08543, USA \\ ${ }^{2}$ The University of Tokyo, Department of Aeronautics and Astronautics, Tokyo 113-8656, Japan
}

\begin{abstract}
Laser-induced incandescence measurements were conducted in the carbon arc discharge, used for synthesis of carbon nanostructures. The results reveal two spatial regions occupied by dominant populations of carbon particles with different sizes. Close to the axis of the arc, large micron size particles dominate the incandescence signal. In the arc periphery, the dominant population of nanoparticles has diameter of $20 \mathrm{~nm}$. Using a heat transfer model between the gas, arc plasma and the particles, it is shown that such a drastic difference in the particle sizes can be explained by evaporation of the micron-scale particles which move across the arc plasma towards the arc periphery. It is also hypothesized that mass evaporated from the micro particles contributes to the carbon feedstock for the formation of nanostructures.
\end{abstract}

\section{Introduction}

An arc discharge with consumed graphite electrodes is commonly used as a synthesis technique for production of quality carbon nanotubes (CNTs) and variety of different 0-D, 1-D and 2-D carbon nanostructures such as fullerenes C60, flakes, carbon nano-wires and nanosheets. This synthesis technique has been studied for a long time; however the growth mechanism is still unclear for both CNTs and other structures. A common approach for evaluation of the nanostructures is their collection on a stationary witness plate during the experiment and a following ex-situ analysis of the structures collected on the plate with material evaluation tools. A more advanced technique is a short time deployment of a tungsten probe, which is then extracted and also analyzed ex-situ [2]. In addition to relying on ex-situ evaluation, stationary and moving probes cannot survive in harsh plasma environments, e.g. the arc between the electrodes, only allowing the inspection of structures far away from the plasma. So far no insitu measurements of nanostructures in a carbon arc were reported and the features of the particles in the arc vicinity remain a mystery.

* Corresponding author, email address : syatom@pppl.gov 
The motivation of this work is to detect nanoparticles in the carbon arc discharge, in-situ. The question of where does the carbon feedstock comes from is very important for consolidation of the picture of the synthesis of single and multiwall carbon nanotubes and other nanostructures in the carbon arc discharge. To that end we employ the laser-induced incandescence (LII) technique, which relies on rapidly heating particles with a laser pulse and observing the resulting incandescence. LII was shown to be a very powerful tool for in-situ measurement of carbon soot particles in the flames. Applying LII model allows deducing the particle size, density and temperature with high temporal and spatial resolution [3]. The application of this technique is also developing in plasma environment and several studies have been published. Several examples include detecting dust particles and determining their temperature [4] and size [5- 7] in low-pressure plasmas. In our work we have applied the LII for the first time, in order to measure nanoparticles size, in situ, in high-pressure plasma environment of carbon arc. We employ an optical setup that allows the investigation of the arc with a spatial resolution down to $0.1 \mathrm{~cm}$. The experiments were conducted using a solid graphite anode, manufactured from a bulk graphite piece. To obtain the distribution of particle sizes from the LII signal, a model that encompasses the arc plasma effects on the temperature of particles was developed and utilized. The interpretation of LII signals obtained in plasma environment involves consideration of plasma effects on the particles temperature that were not previously explored in the combustion environment. We present the mean particle size distribution as a function of radial distance from the arc axis, and discuss possible mechanisms of the formation of detected particles and implications of these results for synthesis of carbon nanotubes.

\section{Experimental and modeling procedure}

\subsection{Experimental setup}

In this work, we employ a carbon arc setup with two cylindrical graphite electrodes, with diameters of $0.55 \mathrm{~cm}$ and $1.15 \mathrm{~cm}$, for anode and cathode, respectively. The electrodes are positioned vertically, with the cathode on top and the chamber is filled with 500 Torr of helium gas. The arc is initiated by bringing the cathode and anode electrodes in contact. Once the arc is ignited, the electrodes are gradually separated, with a stepper motor, to maintain pre-set values of the current and the voltage of $27 \mathrm{~V}$ and $60 \mathrm{~A}$, respectively. The duration of the arc was up to 2 
minutes, in order not to overheat the chamber. In these experiments, we used the LII setup described elsewhere [8], which was modified to introduce a flat-top laser beam profile and the spatially-resolved measurements of particle incandescence. The laser employed is a Quantel Ultra series, a Nd: YAG laser, providing a $1064 \mathrm{~nm}$ laser pulse with a full width at half maximum (FWHM) duration of $\sim 8 \mathrm{~ns}$. The laser beam was shaped and clipped to provide a nearcircular, flat-top profile, with a radius of $0.5 \mathrm{~cm}$ and energy of $25 \mathrm{~mJ}$, resulting in a laser fluence of $0.17 \mathrm{~mJ} / \mathrm{cm}^{2}$. The laser beam was shot in between the electrodes at a frequency of $2 \mathrm{~Hz}$.
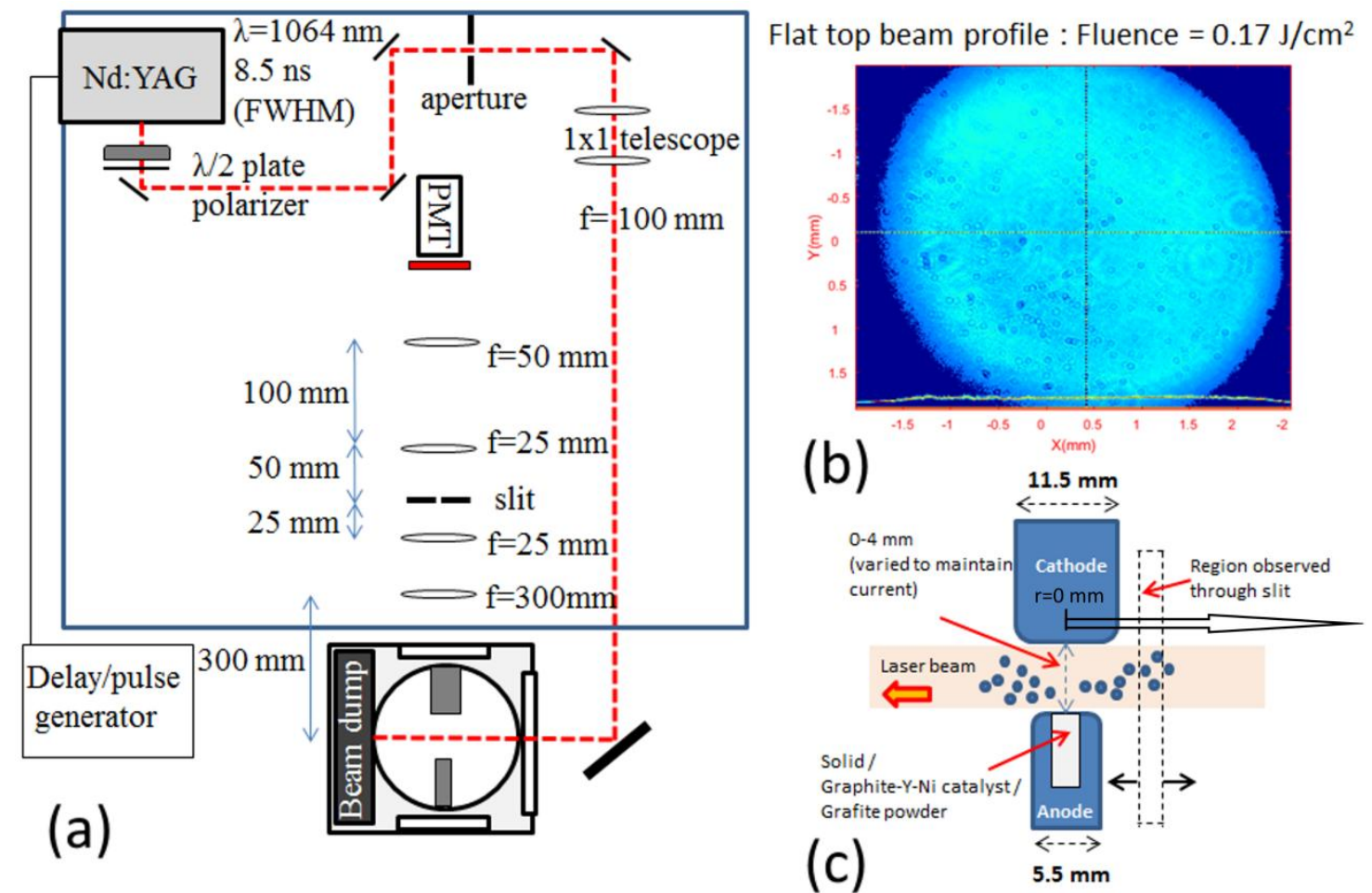

Figure 1. (a) Experimental setup of the arc and the optical arrangement for time-resolved LII measurements (b) "Flat-Top" laser beam profile (c) The arrangement of laser, electrodes and radial scan with slit.

For time-resolved measurements of the LII, we employed an optical setup that images the discharge gap on a plane outside the chamber window. A mechanically controlled slit was used to filter a spatial region of interest of the image and relay it further on to a photomultiplier tube (PMT), equipped with optical filter for $690 \mathrm{~nm}$. The width of the slit and its position were controlled, to manage the width of the observed region and the position with respect to the vertical axis of the arc - in that way we can get the time-resolved LII curve for different 
segments in radial direction. The PMT signal is recorded with a LeCroy WaveSurfer 10 oscilloscope $(1 \mathrm{GHz}, 10 \mathrm{Gs} / \mathrm{s}$ ). The PMT channels on the oscilloscope were loaded to 50 Ohms, which renders the oscilloscope not sensible to a constant-like light (such as radiation from arc) but rather sensible to signals of microsecond and below timescales. The arc light produces a flat waveform, plagued with a high-frequency noise, of almost constant amplitude. To further stress the point: when the PMT observes the arc region, including the hot electrodes, without the prior laser shot - no characteristic LII signal is observed, rather the flat waveform with regular noise. Typical time-resolved (TiRe) LII signal appears when the laser is shot, featuring a rise-time on a timescale of single nanoseconds, due to heating by the laser pulse, followed by the signal decay on a microsecond time-scale, associated with the particle's cooling. The TiRe LII signals were captured at frequency of $2 \mathrm{~Hz}$, during the arc runs of about 100 seconds, resulting in 200 recorded waveforms of TiRe LII signal. A satisfying signal-to-noise ratio (SNR) was obtained with a slit observing a segment of $0.225 \mathrm{~cm}$ in radial direction. We moved the slit in steps corresponding to $0.05,0.1$ and $0.125 \mathrm{~cm}$ for different cases. LII signals were recorded starting from $\mathrm{r}=0.1 \mathrm{~cm}$ and as far as $\mathrm{r}=1.3 \mathrm{~cm}$. For each radial distance, the waveforms with a good $\mathrm{SNR}$ were chosen, and a low-pass filter was applied to remove the high-frequency noise from the constant component of arc light. All chosen signals were peak-normalized and then averaged, so each radial segment has a representative LII curve. The far-from-axis curves look very akin, featuring faster decay of the incandescence when compared to the curves from the vicinity of the arc axis. The most significant change occurs at a radial distance of $0.6 \mathrm{~cm}$ with respect to arc axis. The TiRe LII curves representing different radial segments are shown at Fig.2 (a). Qualitatively speaking, curves that show faster decay represent the cooling of smaller particles, meaning that large particles dominate the incandescence close to the arc axis. However the temperature of the gas and the temperature of the particles also influence the cooling rate and they need to be considered when the analysis of the particle sizes is conducted.

\subsection{Calculation of the particle sizes with LII model}

To obtain the particles sizes distribution with TiRe LII one needs to compare the experimental signal with a signal modeled from a given particle distribution. The models for temporal evolution of LII signal are developed in combustion community for the last two decades and involve the processes that contribute to the energy and mass balance of the particle. 

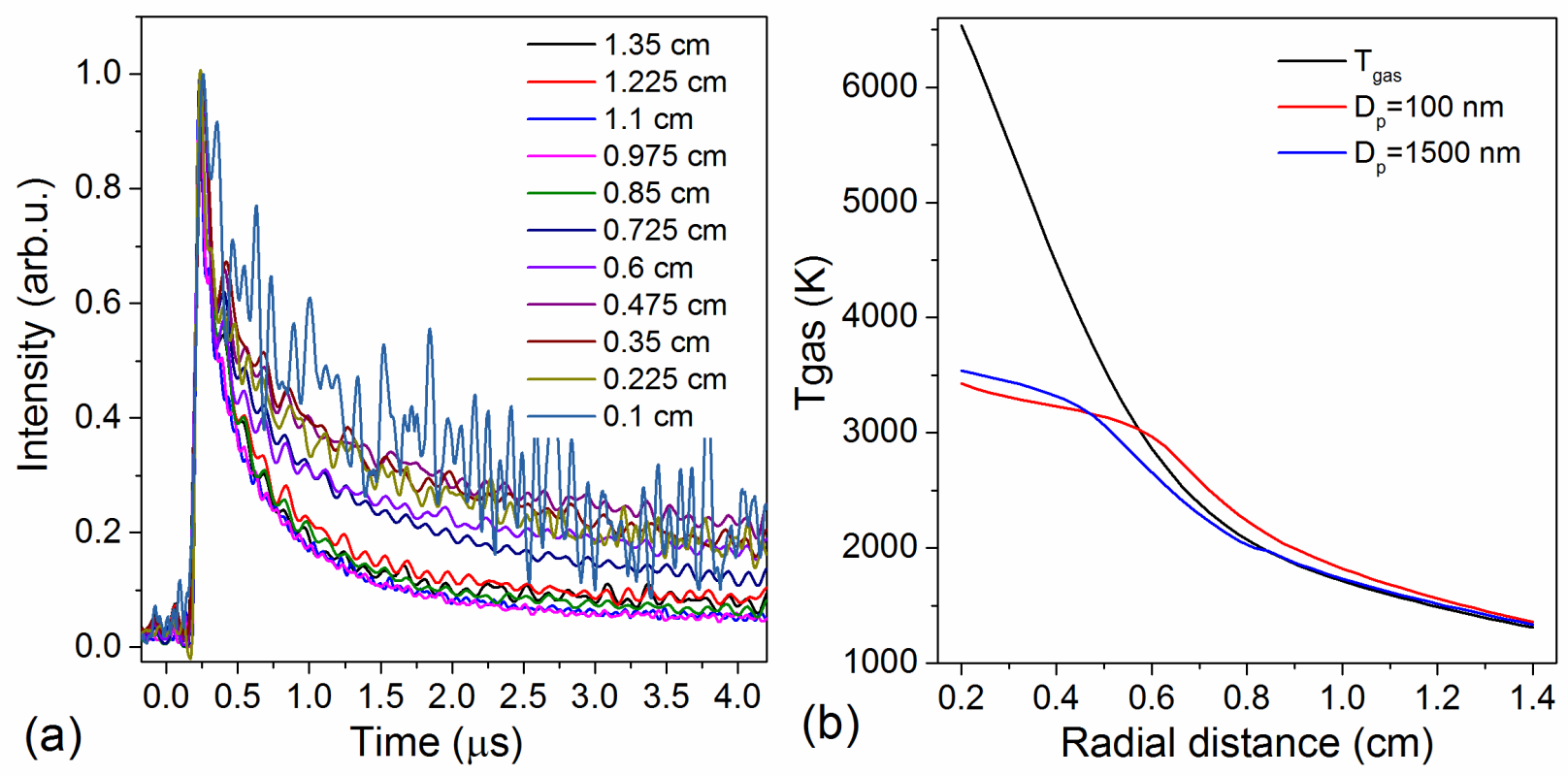

Figure 2. (a) Laser induced incandescence as a function of time for different radial distances with respect to the axis of the arc (solid graphite anode case) (b) Gas temperature from the 2D arc simulation with ANSYS CFX, and examples of particle temperature for two different diameters as a function of radial distance.

Most of recent developments and concerns in the LII modeling are summarized in the review by $\mathrm{H}$. Michelsen et al [3]. The evolution of particle size and diameter is calculated by the solution of the time-dependent mass and energy balance equation. For energy balance, most of the models consider the heat transfer due to laser absorption $\left(\dot{Q}_{a b s}\right)$, conduction $\left(\dot{Q}_{c o n}\right)$, oxidation $\left(\dot{Q}_{o x}\right)$, radiation $\left(\dot{Q}_{\text {rad }}\right)$, sublimation $\left(\dot{Q}_{s u b}\right)$ and thermionic $\left(\dot{Q}_{\text {the }}\right)$ emission. Annealing is usually not taken into account because very little is known about this mechanism, its rate, energetics and effect on physical parameters, such as optical properties [3]. Under arc condition we also consider the role of radiation from the arc plasma $\left(\dot{Q}_{\text {arc }}\right)$ in the heating of the particles [9], and neglect the oxidation effects due to lack of oxygen. Eventually the energy balance equation used for carbon arc environment is:
(1) $\frac{\mathrm{dU}_{\text {int }}}{\mathrm{dt}}=\dot{\mathrm{Q}}_{\mathrm{abs}}+\dot{\mathrm{Q}}_{\mathrm{arc}}+\dot{\mathrm{Q}}_{\mathrm{con}}+\dot{\mathrm{Q}}_{\mathrm{sub}}+\dot{\mathrm{Q}}_{\mathrm{rad}}+\dot{\mathrm{Q}}_{\mathrm{the}}$

The rate of change in the internal energy is expressed as: 
(2) $\frac{d U_{\text {int }}}{d t}=\rho_{s} c_{s} \frac{\pi D_{p}^{3}}{6} \frac{d T}{d t}=M c_{s} \frac{d T}{d t}$

where $\rho_{s}$ - particle density, $c_{s}$ - particle specific heat, $D_{p}$ - particle diameter, $M$ - particle mass and $T$ - particle temperature. The following system of ordinary differential equations for the carbon arc discharge model is then constructed:

(3) $\frac{\mathrm{dT}}{\mathrm{dt}}=\frac{1}{\mathrm{Mc}_{\mathrm{s}}(\mathrm{T})}\left(\dot{\mathrm{Q}}_{\mathrm{abs}}+\dot{\mathrm{Q}}_{\mathrm{arc}}+\dot{\mathrm{Q}}_{\text {con }}+\dot{\mathrm{Q}}_{\mathrm{sub}}+\dot{\mathrm{Q}}_{\mathrm{rad}}+\dot{\mathrm{Q}}_{\text {the }}\right)$, substituting $\mathrm{Eq}(2)$ to $\mathrm{Eq}(1)$.

(4) $\frac{\mathrm{dM}}{\mathrm{dt}}=\left(\frac{\mathrm{dM}}{\mathrm{dt}}\right)_{\text {sub }}$ : mass loss due to sublimation, related to diameter via $D_{p}=\left(\frac{6 M}{\pi \rho_{s}}\right)^{\frac{1}{3}}$.

(5) $\frac{d q_{p}}{d t}=\pi D^{2} \int_{0}^{t} J_{\text {the }}(s) d s$ : electrical charging rate, due to thermionic emission [10].

The system of equations is solved by the Runge-Kutta $4^{\text {th }}$ order scheme, to obtain the particle charge, mass, diameter and charge. The LII signal is calculated using the Plank function, modified by emissivity $\epsilon$ :

(6) $\mathrm{S}=\epsilon \frac{2 \pi^{2} D_{p}{ }^{2} h c^{2}}{\lambda_{\mathrm{S}}^{5}\left[\mathrm{e}^{\mathrm{hc} / \lambda_{\mathrm{s}} \mathrm{k}_{\mathrm{B}} \mathrm{T}}-1\right]}$

A brief, but more detailed description of the calculation for all $\dot{Q}$ terms can be found in the appendix for this paper. Even though the Rayleigh approximation is commonly used to calculate the particles' emissivity due to its convenience under the conditions $D_{p} \ll \lambda_{l}$, where $\lambda_{\mathrm{l}}$ is the laser wavelength, in the carbon arc discharge where the primary particle size can be micron size as well, the Rayleigh approximation is no longer valid. Therefore, the Mie theory is used on the calculation for the heating rates: $\dot{Q}_{a b s}, \dot{Q}_{a r c}, \dot{Q}_{\text {rad }}$ and the LII signal, $S$. Details for Mie calculation are given in the appendix.

The LII model is employed to calculate a simulated LII signal from an arbitrary distribution of particles. The input distribution is modified until the simulated and the experimental signals are as close as possible to be identical. Other two important inputs for the model are the gas temperature and the initial temperature of the particles, before they are heated by the laser. To obtain radial gas temperature profile a 2D-axisymmetric simulation of carbon arc discharge in helium atmosphere was performed, with arc model based on a hydrodynamic approach [2], with 
rigorous treatment of heat production and heat and species transfer. Electron and heavy particles temperatures were treated separately. Heat conduction by plasma, gas, and electrodes was considered, as well as mutual radiation of electrodes. All computations were performed with general purpose CFD-code ANSYS CFX, which was customized in order to implement the arc model. Detailed results of the simulation will be published in a separate paper. Knowing the gas temperature we can now calculate the temperatures of the particles. When the particles are injected into a buffer gas of a known temperature they will either cool down or heat up to a certain temperature value. We calculate this temperature for each particle size and several injection temperatures $\left(1000 \leq T_{\text {injection }} \leq 4000 \mathrm{~K}\right)$, when all of the heating and cooling mechanisms described earlier are accounted for, with the exception of the laser absorption. The converged particle temperatures for two exemplary sizes are shown at Fig.2(b), as a function of radial distance. Close to the arc axis, where the gas is very hot, the "converged" temperature of the particle is significantly lower than the gas temperature, due to evaporative cooling. In the cooler regions the temperature of the particles becomes a little bit higher than the gas temperature owing to the radiative heating from the arc. The time that it takes for the particle temperature to converge depends on the gas temperature and the particle size. Exemplary results of particle temperature convergence are shown at figure 3, for particles sizes of 1000 and 100 $\mathrm{nm}$, injected into gas with temperature of 7000 and $3000 \mathrm{~K}$. Large particles "thermalize" on longer times, that grow even longer as the gas temperature decreases. The time range for "thermalization" of particles with diameters of 10-2000 nm and gas temperatures between 1000$8000 \mathrm{~K}$ is $1-80 \mu \mathrm{s}$. Is this "thermalization" time short enough? To answer this question we can estimate the residence time of a particle in a radial segment of $0.2 \mathrm{~cm}$, which is the scale of the region from which we collect the LII signal. Large particles with diameters on micron scale possess flow velocities. The hydrodynamic ANSYS CFX simulation described earlier yields maximum flow velocities $<10 \mathrm{~m} / \mathrm{s}$ and actually one can evaluate flow velocities in the interelectrode gap, without the complex simulations. Fluid motion in the inter-electrode gap is caused by the flow of gaseous material due to the ablation of anode. Fluid velocity $\vec{v}$ can be obtained from fluid mass flux $\vec{g}$ and density $\rho$, via $\vec{v}=\frac{\vec{g}}{\rho}$. To get the upper limit for the flow velocity we calculate the lower limit for gas density, based on ideal gas approximation: $\rho_{\min }=\frac{p \cdot \mu_{H e}}{R \cdot T_{\max }}=$ $0.004 \frac{\mathrm{kg}}{\mathrm{m}^{3}}$, where $p=68 \cdot 10^{3}$ is the chamber pressure, $\mu_{H e}=4 \frac{\mathrm{g}}{\mathrm{mol}}$ - helium molar mass (for 
upper limit we assume all gas is the light helium), $R=8.31 \frac{\mathrm{J}}{\mathrm{mol} \cdot \mathrm{K}}-$ gas constant and $T_{\max }=8000 K-$ highest temperature in the arc region.
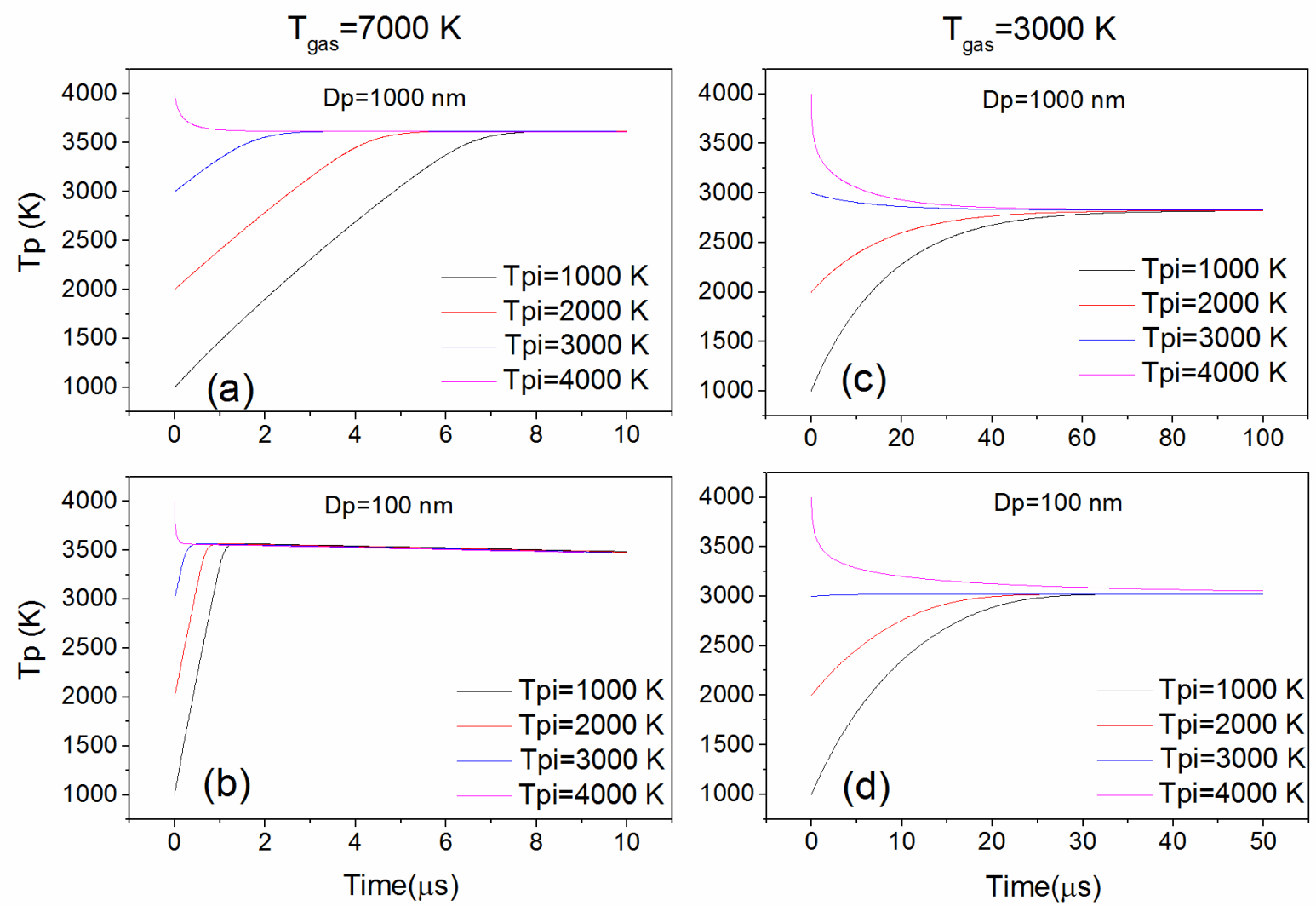

Figure 3. Time evolution of temperature of the particles injected with initial temperatures of 1000-4000 K. (a) Particle diameter $-1000 \mathrm{~nm}, \mathrm{~T}_{\mathrm{gas}}=7000 \mathrm{~K}$ (b) Particle diameter -100 $\mathrm{nm}, \mathrm{T}_{\mathrm{gas}}=7000 \mathrm{~K}$ (c) Particle diameter $-1000 \mathrm{~nm}, \mathrm{~T}_{\mathrm{gas}}=3000 \mathrm{~K}$ (d) Particle diameter $100 \mathrm{~nm}, \mathrm{~T}_{\text {gas }}=3000 \mathrm{~K}$.

Fluid mass flux can be approximated as: $g \approx \frac{G_{a}}{\pi r_{a}^{2}}$, where $G_{a}=1 \frac{\mathrm{mg}}{\mathrm{s}}$ is the ablation is rate and $r_{a}=0.275 \mathrm{~cm}$ is the radius of the anode. This relation is based on the assumption that fluid mass flux in the inter-electrode gap is equal to ablation flux of carbon from the anode surface. We assume that mass flux of helium in the inter-electrode gap is negligible compared to that of carbon. Helium doesn't flow in between the electrodes, being unable to flow from and into the carbon electrodes and this behavior was also observed in the simulation. According to the abovewritten formulae, the upper limit for fluid velocity in the inter-electrode gap is: 
$v=\frac{G_{a}}{\pi r_{a}^{2}} \cdot \frac{1}{\rho_{\min }}=\frac{10^{-6} \frac{\mathrm{kg}}{\mathrm{s}}}{0.004 \frac{\mathrm{kg}}{\mathrm{m}^{3}} \cdot \pi \cdot(0.00275 \mathrm{~m})^{2}} \approx 10.5 \frac{\mathrm{m}}{\mathrm{s}}$

When particle sizes are smaller than the mean free path $\left(\lambda_{m f p}=\frac{1}{\sqrt{2} \sigma_{c} n_{g}} \approx 1 \mu \mathrm{m}, \sigma_{c}-\right.$ collisional cross section, $n_{g}$ - gas number density) of carbon atoms and molecules- these particles are essentially frictionless. It is assumed here that velocities of such particles are determined by their temperature: at $T_{\text {gas }}=7000 \mathrm{~K}$ small particles of diameter $<10 \mathrm{~nm}$ will approach velocities of $10 \mathrm{~m} / \mathrm{s}$, while the larger particles will be slower. Hence, assuming the flow velocity of $10 \mathrm{~m} / \mathrm{s}$, the minimal residence time of particles in $0.2 \mathrm{~cm}$ radial segment is $\sim 200 \mu \mathrm{s}$. Since the maximum temperature convergence time is $\sim 80 \mu \mathrm{s}$, we can safely assume that all particles in the volume defined by the slit have converged temperatures that we have calculated.

In the fitting process for each curve recorded at a certain radial distance, we introduce a distribution of particle diameters, gas temperature, and calculate the corresponding temperature of each particle with a given diameter. Then, the LII curve is calculated and compared to the experimental curve. The particle sizes are adjusted (and their temperatures are adjusted accordingly), until a best fit between the calculated and experimental curves is obtained. It is important to note that micron-scale particles dominate the LII signal, even if greatly outnumbered by nano-scale particles (up to $\sim 3$ orders of magnitude). This means that a presence of a single large particle will be definitely noted in a "great sea" of small particles but not the other way around. Thus, the LII signal will be dominated by large particles if these are present. Figure 4 shows the comparison of the experimental LII and the best fit modeled LII for $\mathrm{r}=0.225$ and $\mathrm{r}=1.25 \mathrm{~cm}$ ( $\mathrm{r}$ - radial distance from arc axis). The particle temperature has a strong influence on the decay rate of LII signal and the particle diameter sets the level of the "plateau" to which the signal falls, so there is no possibility that two very different sets of temperatures and particle diameters will yield similar LII curves. This implies that these two parameters have a unique set for each fitted curve, however small $(<10 \%)$ variation of each parameter is acceptable and is factored in the error estimation. The best fit is obtained with lognormal distributions, where the standard deviation is close to $50 \%$ of the mean value (see the inserts at Fig.4). It is worth noting that the distributions with micro-particles neglect the possibility of nanoparticles being present too. As we have described earlier- the micro-particles, even if greatly outnumbered by nanoparticles, will dominate the LII pattern. The contribution of nanoparticles will be hardly 
noted in this case. That means that significant nanoparticle densities can be found in the regions where LII modeling shows micro-particle distributions. Lognormal distribution is easy to control and vary; it has no tail in the small-diameter region and yields very good fits, especially for LII signals in the inter-electrode gap.
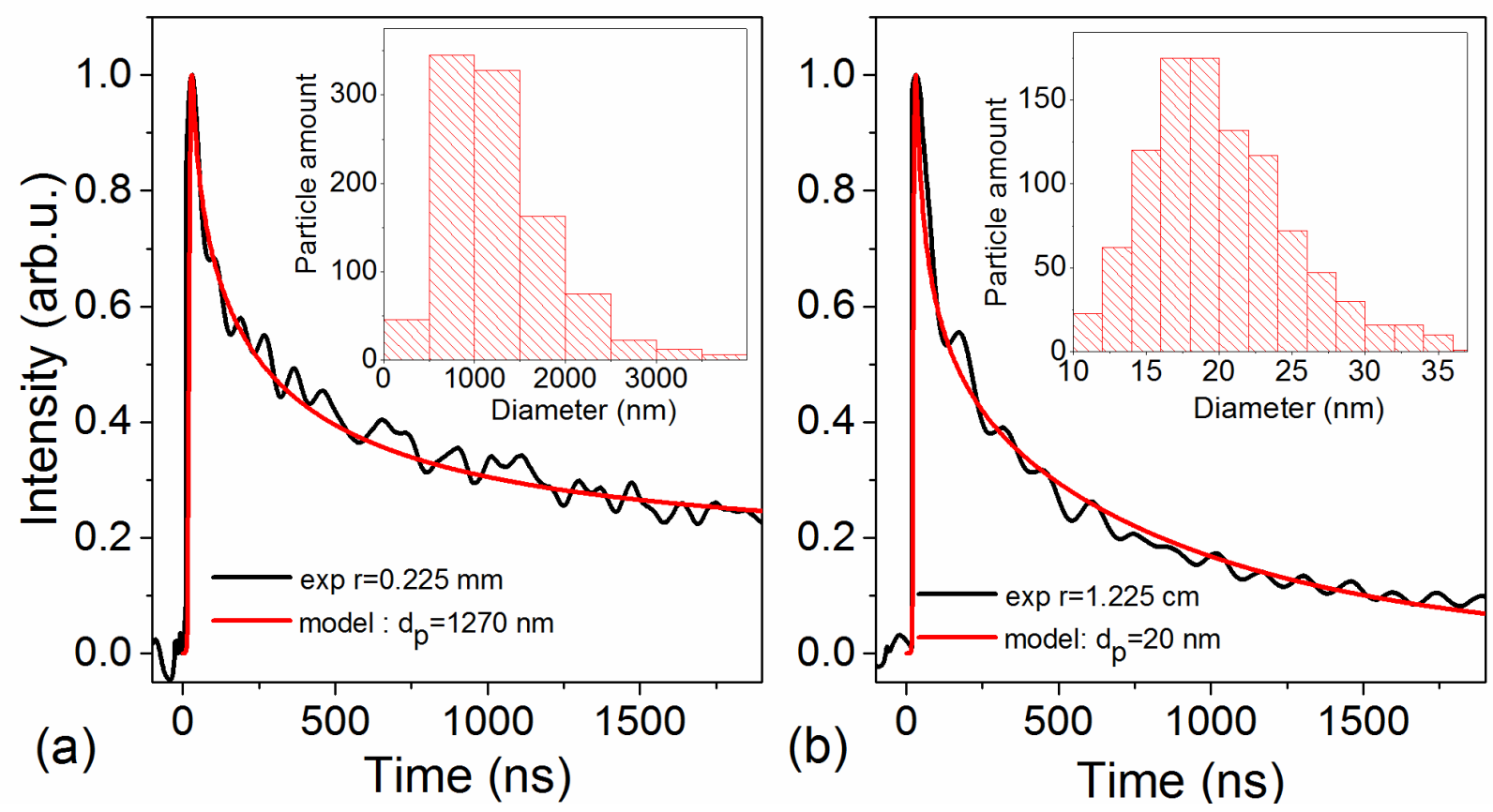

Figure 4. Examples of experimental and fitted curves for solid anode case (a) $\mathrm{r}=0.225 \mathrm{~cm}$ and (b) $\mathrm{r}=1.225 \mathrm{~cm}$. The inserts show the polydiameter particle distribution for the fit.

We should note that the cooling due to thermal diffusion to the interior of the particle was neglected in our calculations. Such effect (thermal wave effect) is not important in small particles but it might have an effect if the particle is large (micron-scale). Looking at the decays curves we expect the larger particles to be located closer to the axis of the arc. We assume that these particles are graphite chunks, ablated from the anode, since no synthesis can possibly take place in such a hot region of the arc. Let's consider a large particle with diameter $D_{p}=1.2 \mu \mathrm{m}$, located in the hottest part of the arc with $T_{\text {gas }}=7000 \mathrm{~K}$. As it can be seen from figure $3(\mathrm{a})$, the particles temperature will be $\sim 3500 \mathrm{~K}$. Typical time for thermal diffusion in radial direction is: $\tau_{\text {diff }}=\frac{\mathrm{R}_{\mathrm{p}}^{2}}{\chi}=18.5 \mathrm{~ns}$ where $\mathrm{R}_{\mathrm{p}}$ is the particle radius and $\chi=\frac{\kappa}{\rho c_{\mathrm{p}}}$, with $\kappa \sim 0.304 \frac{\mathrm{w}}{\mathrm{cm} \cdot \mathrm{K}}$ [11] being the thermal conductivity at $\mathrm{T}=3500 \mathrm{~K}, \rho \sim 2.3 \frac{\mathrm{g}}{\mathrm{cm}^{3}}$ the mass density and $\mathrm{c}_{\mathrm{p}} \sim 0.7 \frac{\mathrm{J}}{\mathrm{g} \cdot \mathrm{K}}$ the 
specific heat capacity of graphite. Obviously, since this time is proportional to the squared radius, it will be much shorter for smaller diameters. The nanosecond time scale for thermal diffusion is very fast compared with the timescale of the observed cooling, which happens on scale of 1-2 microseconds (Fig.2a). This allowed us to make a quasi-adiabatic assumption that the interior and the exterior of the particle is in equilibrium (i.e. at the same temperature) during the cooling phase of the particle. In the other words, the LII signal is not affected by the thermal diffusion

Note that, the thermal diffusion can however change the particle temperature on the short time scale, during and immediately after the laser pulse. We would like to remind that the initial particle temperature is calculated from the gas temperature (see earlier discussion and figure 3). We use the calculated particle temperature as an initial input for the fitting and change it to obtain the best fit. Tailoring the particle temperature this way will also account for the initial change due to thermal diffusion to the unheated interior. This variation of the particle temperature is also factored into the error estimation.

\section{Discussion}

Figure 5 shows the mean size of the particle distribution for different radial segments. The particles close to the axis have sizes on the scale of micron. The sizes gradually decrease up to $\mathrm{r}=0.6 \mathrm{~cm}$ where a sharp decrease in the size is observed. At $\mathrm{r}=0.6 \mathrm{~cm}$ (which is also the edge of the cathode) the particles emerge with sizes $<30 \mathrm{~nm}$. The picture apparent from the LII results is as follows. In the vicinity of the arc axis, micron-sized particles are present and they gradually lose size due to sublimation. Up to the distance of $0.5 \mathrm{~cm}$ from the arc axis, the mean particle size gradually decreases loosing approximately $55 \%$ of the initial size. Outside the interelectrode gap dominant particles are nanoparticles with the mean diameter of below $30 \mathrm{~nm}$, entailing a mean size drop by factor larger than 10 , on a length of $0.1 \mathrm{~cm}$. Beyond $\mathrm{r}=0.6 \mathrm{~cm}$, there is no significant change in the mean size, up to the distance of $1.3 \mathrm{~cm}$ from the arc axis. Further away from the arc, no LII signal is detected, which can be interpreted as a decrease of the volume density of the nanostructures below a LII detection threshold. 


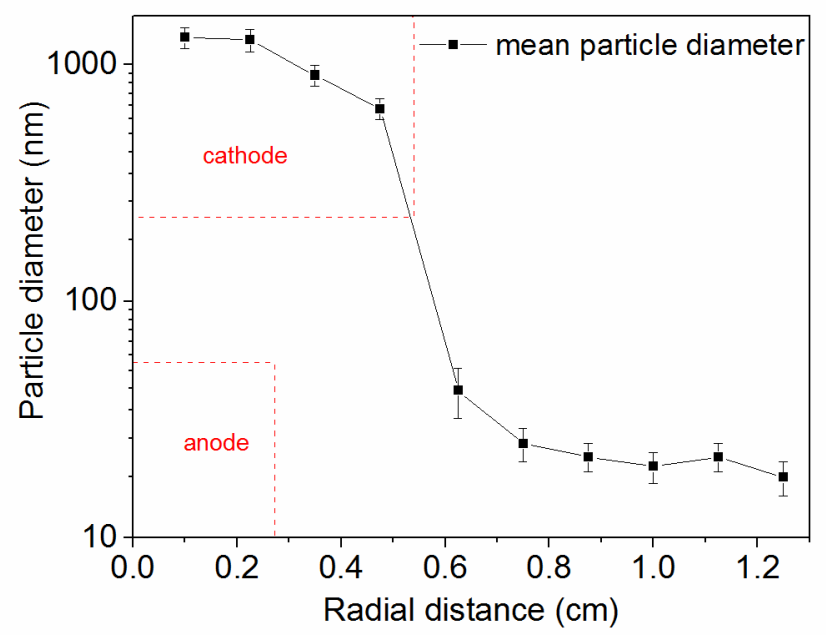

Figure 5. Diameter of the particles calculated from LII versus radial distance from the axis of the anode.

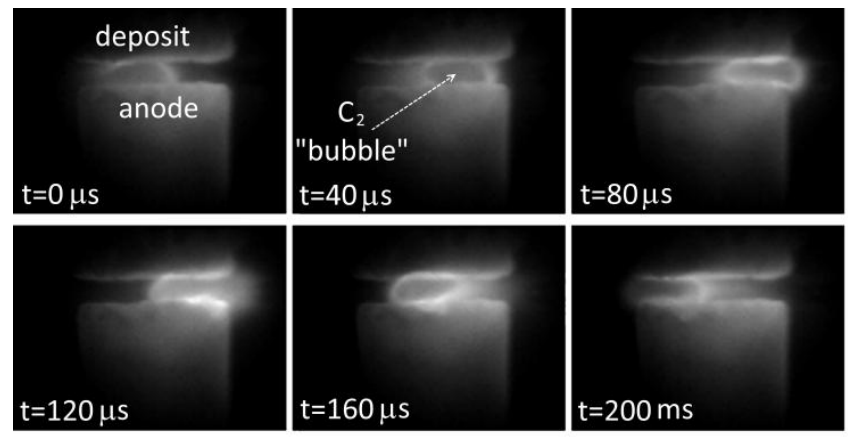

Figure 6. Sequence of images depicting the fast rotation and oscillation of the arc.

We shall now ponder the question of where the micron-diameter particles come from. It is well known that graphite ablation by laser pulses generates micron-sized particles [12]. The results were obtained while applying laser fluences starting from $10 \mathrm{~J} / \mathrm{cm}^{2}$ and up to $5000 \mathrm{~J} / \mathrm{cm}^{2}$. We propose that in arc discharge, the ablation of the anode can also yield particles with diameters in micron range, due to a rapid heating by the arc, due to arc oscillations in a $\mathrm{kHz}$ frequency range. The fast movement of the plasma channel causes an uneven heating of the anode surface, creating thermal tension and fracturing of the material on a large scale, which eventually induces the release of large graphite chunks or clusters. Low frequency arc oscillations ( $100 \mathrm{~Hz})$ [13] and high frequency oscillations ( $1 \mathrm{kHz})$ [14] were previously reported. Fig. 6 depicts the $\mathrm{kHz}$ oscillations via a sequence of images, acquired with a fastframing camera equipped with a $470 \mathrm{~nm}$ optical filter to monitor the $\mathrm{C}_{2}$ molecular emission in the 
arc. One can see the apparent "bubble-like" distribution of $\mathrm{C}_{2}$ and it is evident on the pictures that the bubble moves from side to side. A less apparent observation is that the bubble not merely moving from side to side, but rather is rotating around the anode surface. The rotation is easily recognizable in a video-sequence and less so from a few images. However, if we take a look at the images in the bottom row of figure 5, we can see a bright area where the "bubble" touches the anode. This bright anode "spot" is evident when the arc is close to the front side (i.e. the side facing the camera) of the anode and when the arc in on the opposite side of the anode- the spot is not observed. The approximate time that takes the arc to complete a single revolution around the arc is $200 \mu \mathrm{s}$. We can compare the conditions in the arc and the laser ablation experiment [12], by calculating the "plasma fluence" equivalent to the laser fluence. We assume that the heat is delivered to the anode through the anode spot, which is the area of the plasma channel attachment to the anode, with radius of $\sim 0.05 \mathrm{~cm}$ [15]. To estimate the energy flux rate to the anode we use [15]: $\dot{q}_{a} \approx \frac{I_{a r c} \times V_{e f f}}{a_{a s}}$, where $a_{a s}$ stands for the area that contains the current, i.e. the area of the anode spot $\left(\sim 0.008 \mathrm{~cm}^{2}\right), I_{\text {arc }}$ is the arc current of $60 \mathrm{~A}$ and $V_{e f f}$ is the volt equivalent of the heat flux, defined as the ratio of total heat flux to the anode to the arc current $(\sim 11 \mathrm{~V}$ for a $0.6 \mathrm{~cm}$ diameter anode, [15] ). To obtain an equivalent value to fluence, we multiply $\dot{q}_{a}$ by the characteristic time that the arc spends in the surface area equal to the anode spot $(\approx 30 \mu s)$ and obtain $\sim 3 \mathrm{~J} / \mathrm{cm}^{2}$. This calculation suggests that it is not unreasonable to expect similarity between the ablation by an unstable arc and laser driven ablation, e.g. the generation of micro-particles in arc discharge.

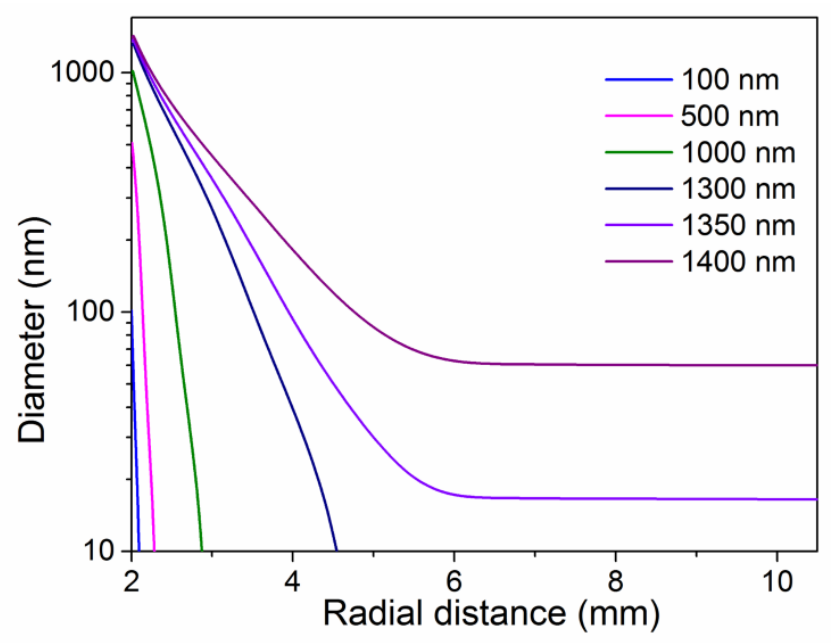


Figure 7. Size loss of different particles traveling in radial direction with velocity of $5 \mathrm{~m} / \mathrm{s}$.

Particles are injected at radial location of $\mathrm{r}=0.2 \mathrm{~cm}$.

Another interesting question is what causes such a sharp separation to two distinct populations: large (>500 nm) particles in the inter-electrode gap and $\sim 20 \mathrm{~nm}$ particles outside. To that end we perform a simulation, employing the particle heat transfer model described earlier. In the simulation, we release particles at $\mathrm{r}=0.2 \mathrm{~cm}$, with a radial velocity of $5 \mathrm{~m} / \mathrm{s}$ and track the change of the particle size due to all the relevant mechanisms apart from the laser heating. The arc position is fixed to $\mathrm{r}=0$ and the arc channel width is set to $0.2 \mathrm{~cm}$. The particles are released outside the arc channel and close to the arc boundary. The results of the simulation on Fig 7, imply that the particles have to be larger than 1.3 microns in order to get out of the inter-electrode gap. Smaller particles evaporate completely before escaping the hot region. The size decay rate for $\mathrm{r}<0.6 \mathrm{~cm}$ is not uniform; it is slower for larger sizes and slightly faster for sizes below 300-400 $\mathrm{nm}$. The change of the slope is not as evident as changes observed in the experimental results. This could be because the actual behavior of the arc is unstable (see Ref.[13,14] and the earlier discussion). In particular, the arc attachment to the anode and the arc column move between the electrodes affecting the emittance of the particles from the anode and the anode ablation. The arc rotation (see Fig. 6 and the following discussion) may also add a tangential component to the particle velocity, altering to the residence time of particles in the hot zone and their subsequent evaporation. Finally, the model doesn't consider that particles will have velocities that change with location and temperature. To account for all these factors one needs to employ more complex models and simulation, which are out of the scope of this work. Let us note that the picture of particles evaporation as a function of radial distance, as shown at Fig. 7 for $v=5 \mathrm{~m} / \mathrm{s}$, is sensitive to the particle velocity. For example with $\mathrm{v}=3 \mathrm{~m} / \mathrm{s}$ even particles with diameter of 1.5 micron will evaporate completely within $0.1 \mathrm{~cm}$ and the particle needs to be 2 micron large to evade the arc region, while with $\mathrm{v}=10 \mathrm{~m} / \mathrm{s}$ particles with diameter $900 \mathrm{~nm}$ already emerge with from the hot region. However in the high velocity case large particles of different diameters will escape the cathode-anode gap with very different diameters, unless there is some other mechanism, that we are unaware of, equalizing the sizes outside the arc. Since we detect a fairly universal distribution of nanoparticles outside the gap we can conclude that there are no micron particles emitted from the anode with velocities v $>8 \mathrm{~m} / \mathrm{s}$. Nevertheless, our model predicts the massive evaporation of large particles in the arc. Outside the arc core we observe a 
nearly uniform size of the nanoparticles, countering the idea that these are particles which managed to escape from the arc. Therefore, we hypothesize that nanoparticles measured outside the arc were also formed outside the arc, by clustering of carbon atoms and molecules. At least a part of these atoms and molecules is supplied by the sublimation of the micro-particles.

Last point that deserves consideration is the shape of the structures in the inter-electrode gap. The rates of the heating process for spherical particles by radiation are dependent on the particle's volume, but the most important cooling processes (conduction and evaporation) are proportional to the surface area. This makes it possible to estimate the size of the particles on the basis of the LII signal. We observe a LII signal in the inter-electrode gap and interpret it as a signal from micron-sized, spherical particles. Generally, treating volumetric non-spherical particle, with random special orientation as spherical particles can be justified to some extent. In the case of our experiment this would change the sizes quantitatively, but not qualitatively, i.e. large formations with micron-dimensions are still ablated from the anode. But what if the formations that produce that signal are actually highly non-spherical? In this case the error could be potentially significant. The heating and cooling processes for planar structures are proportional to their surface area, regardless of their orientation. Therefore, for two-dimensional structures it is very difficult to get reasonable information about the size, based only on the interpretation of the LII signal. Bottom line is the closer the ablating structures are to a $2 \mathrm{D}$ structure - the larger the error of the size evaluation will be. Unfortunately as mentioned above the LII signal of a planar flake cannot be readily related to the size of the flake hence we cannot estimate how much the flake size would different from the size we have calculated under the assumption that the micro-particles are spherical. However, ultimately independent on the shape of detected particles our results demonstrate that the ablation from the anode is not entirely on the molecular and atomic level, but by large particles as well.

\section{Conclusions}

Laser induced incandescence was successfully applied to measure size of nanostructures generated in the carbon arc, for different distances from the arc axis. The conventional LII model [3] was augmented with the contribution of plasma radiation and utilized for interpretation of the experimental results. Our measurements revealed two spatially separated particle groups. Inside 
the inter-electrode gap, we detect micro-particles, with minimal sizes of $\sim 500 \mathrm{~nm}$, with the mean size decreasing as a function of distance from the axis of the arc. Outside the inter-electrode gap, the detected particles have almost a uniform size distribution, around $20 \mathrm{~nm}$, independent of the distance. We propose that micro-particles are produced due to a rapid heating of the anode surface, resulting from the $\mathrm{kHz}$ oscillations of the arc. These particles evaporate in the hot region, rapidly losing their size and are unable to escape the inter-electrode gap. The evaporated mass contributes to the production of the feedstock that forms the nanostructures in the cooler region. In contrast to these micro-particles, the nanoparticles outside the gap are likely produced by synthesis process in the arc plasma.

\section{Appendix: description of the terms in LII model}

\section{Laser absorption}

$$
\dot{Q}_{a b s}=C_{a b s} F q(t) / q_{1}
$$

$C_{a b s}$ is the Mie cross-section, which is given by $\frac{1}{4} \pi D^{2} K_{a b s}$, where $K_{a b s}$ is Mie efficiency for absorption. $F$ is the laser fluence, $q(t)$ is the laser temporal profile used in the research, peaknormalized to unity and $q_{1}$ is the normalization constant for the integrated laser temporal profile.

\section{Arc plasma radiation}

Arc plasma radiation is adopted from the model of the stationary arc discharge given by M. N. Shneider [9]. One thing modified from his model is that the Rayleigh approximation is replaced with Mie regime calculation.

$$
\dot{Q}_{a r c}=\zeta \frac{r_{0}^{2}}{r^{2}} \int_{0}^{\infty} \frac{\pi D^{2}}{4} K_{a b s} \frac{2 \pi h c^{2}}{\lambda^{5}\left(e^{h c / \lambda k_{B} T}-1\right)} d \lambda,
$$

where the arc emissivity $\zeta=0.8$ [9], $r_{0}$ is the arc radius, $r$ is the distance from the arc, $h$ is the Planck constant, $c$ is the speed of light, and $k_{B}$ is the Boltzmann constant.

\section{Conduction}

We distinguish between the transition regime and the free molecular flow regime by the Knudsen number. In the carbon arc discharge situation, both regimes can realize, depending on the particles temperature and size. 
The conductive term in the free molecular regime is calculated by, [3]

$$
\dot{Q}_{c o n, F M}=\frac{\pi D^{2} \alpha_{T} p_{0}}{2 T_{0}} \sqrt{\frac{R_{m} T_{0}}{2 \pi W_{0}}} \frac{\gamma+1}{\gamma-1}\left(T-T_{0}\right)
$$

Here, the thermal accommodation coefficient $\alpha_{T}$ for He is set to 0.13 [16] and $p_{0}$ is ambient pressure, $T_{0}$ is ambient temperature, and $W_{0}$ is the molecular weight of He.

For the transition regime, the following equation is used [17]:

$$
\dot{Q}_{c o n, T R}=\frac{2 \kappa_{a} \pi D^{2}}{D+\lambda_{M F P} G}\left(T-T_{0}\right)
$$

where $\kappa_{a}$ is the thermal conductivity of the ambient gas, $\lambda_{\mathrm{MFP}}$ is the mean free path given as $\frac{1}{\sqrt{2} \sigma_{c} n_{g}}$ ( here $\sigma_{c}$ is collisional cross section, and $n_{g}$ is gas number density) [17], and $G$ is a function of heat capacity ratio given by,

$$
G=\frac{2(9 \gamma-5)}{\alpha_{T}(\gamma+1)}
$$

This transition regime equation is the McCoy and Cha model [18] widely used in the LII community [3, 17]. Thermal conductivity of helium is taken from Ref. [19].

\section{Sublimation}

The expression for the evaporative cooling rate used in the majority of LII models [3] is adopted, and which is given by,

$$
\dot{Q}_{s u b}=-\frac{\Delta H_{v}}{W_{v}} \frac{d M}{d t}
$$

where $\Delta H_{v}$ and $W_{v}$ are the enthalpy of formation and the average molecular weight of the sublimed carbon cluster, respectively. The mass loss of the particle due to sublimation [20] is expressed as 


$$
\frac{d M}{d t}=-\frac{\pi D^{2} W_{v} \alpha_{M} p_{v}}{R_{p} T}\left(\frac{R_{m} T}{2 W_{v}}\right)^{0.5}
$$

where $\alpha_{M}$ is the mass accommodation coefficient, $p_{v}$ is the average saturation partial pressure of the sublimed carbon clusters, and $R_{p}$ and $R_{m}$ are the universal gas constant in different units. Values of each parameter are taken from Table 3 of Ref. [20].

\section{Radiation}

The Plank function is used to calculate the rate of radiative cooling, and the Mie theory is used on calculation as well.

$$
\dot{Q}_{\text {rad }}=\pi D^{2} \int_{0}^{\infty} K_{a b s} \frac{2 \pi h c^{2}}{\lambda^{5}\left(e^{h c / \lambda k_{B} T}-1\right)} d \lambda .
$$

\section{Thermionic emission}

The adjusted thermionic emission model [9], which considers the positive charge buildup in a laser-heated particle is used :

$$
\dot{Q}_{\text {the }}=\frac{(\phi+\Delta \phi) \pi D^{2}}{e} J_{t h e},
$$

here $\phi$ is the work function, $\Delta \phi$ is the increased barrier due to the positive charge buildup, $e$ is the electron charge, and $J_{\text {the }}$ is the adjusted Richardson-Dushman equation [9], which is given as following:

$$
J_{\text {the }}=A_{0} T^{2} \exp \left(-\frac{(\phi+\Delta \phi)}{k_{B} T}\right)
$$

where $A_{0}=4 \pi m_{e} k_{B}^{2} e / h^{3}$ is the Richardson constant.

\section{Mie regime}

It can be shown [21] that for a spherical particle, the emissivity equals to absorption efficiency, $K_{a b s}$. According to the Mie theory [21], the extinction, the scattering and the absorption efficiency can be expressed as,

$$
K_{e x t}=K_{s c a}+K_{a b s}
$$




$$
\begin{gathered}
K_{s c a}=\frac{2}{x^{2}} \sum_{n=1}^{\infty}(2 n+1)\left(\left|a_{n}\right|^{2}+\left|b_{n}\right|^{2}\right), \\
K_{e x t}=\frac{2}{x^{2}} \sum_{n=1}^{\infty}(2 n+1) \operatorname{Re}\left(a_{n}+b_{n}\right), \\
K_{a b s}=K_{e x t}-K_{s c a} .
\end{gathered}
$$

The Mie coefficients, $a_{n}$ and $b_{n}$, are given by

$$
\begin{gathered}
a_{n}=\frac{\mu m^{2} j_{n}(m x)\left[x j_{n}(x)\right]^{\prime}-\mu_{1} j_{n}(x)\left[m x j_{n}(m x)\right]^{\prime}}{\mu m^{2} j_{n}(m x)\left[x h_{n}^{(1)}(x)\right]^{\prime}-\mu_{1} h_{n}^{(1)}(x)\left[m x j_{n}(m x)\right]^{\prime}} \\
b_{n}=\frac{\mu_{1} j_{n}(m x)\left[x j_{n}(x)\right]^{\prime}-\mu j_{n}(x)\left[m x j_{n}(m x)\right]^{\prime}}{\mu_{1} j_{n}(m x)\left[x h_{n}^{(1)}(x)\right]^{\prime}-\mu h_{n}^{(1)}(x)\left[m x j_{n}(m x)\right]^{\prime}}
\end{gathered}
$$

where the complex refractive index $m=2.0-0.1 i$ is used, which is an average over the wavelength range $350-1000 \mathrm{~nm}$ taken from Ref. [22], $x$ is the size parameter which is represented by the multiple of $k=2 \pi / \lambda$, the wave number, and $a$, the radius of the particle. $\mu$ is the magnetic permeability of the ambient medium, $\mu_{1}$ is the magnetic permeability of the sphere, and $j_{n}(x)$ is spherical Bessel function of order $n$. For calculation of the Mie efficiencies and coefficients, the code by C. Mätzler [23] is utilized.

\section{Acknowledgement}

The authors would like to thank B. C. Stratton, M. Schneider, V. Vekselman and J. Mitrani, for very useful suggestions and discussions and to A. Merzhevskiy for technical assistance. This work was supported by the U.S. Department of Energy, Office of Science, Basic Energy Sciences, Materials Sciences and Engineering Division.

\section{References}

[1] N. Arora, N.N. Sharma, Arc discharge synthesis of carbon nanotubes: Comprehensive review, Diamond \& Related Materials, 50 (2014) 135-150. 
[2] X. Fang, A. Shashurin, G. Teel, M. Keidar, Determining synthesis region of the single wall carbon nanotubes in arc plasma volume, Carbon 107 (2016) 273-280.

[3] H. A. Michelsen, C. Schulz, G.J. Smallwood, S. Will, Laser-induced incandescence: Particulate diagnostics for combustion, atmospheric, and industrial applications, Progress in Energy and Combustion Science, 51 (2015) 2-48.

[4] W. W. Stoffels ,E. Stoffels, G. M. W. Kroesen and F. J. de Hoog, Detection of dust particles in the plasma by laser-induced heating, J. Vac. Sci. Technol. A, 14 (1996) 588-594.

[5] G. S. Eom, C. W. Park, Y. H. Shin, K. H. Chung, S. Park, W. Choe and J. W. Hahn, Size determination of nanoparticles in low-pressure plasma with laser-induced incandescence technique, Appl. Phys. Lett., 83 (2003) 1261-1263.

[6] F. M. J. H. van de Wetering, W. Oosterbeek, J. Beckers, S. Nijdam, E. Kovačević and J. Berndt, Laser-induced incandescence applied to dusty plasmas, J. Phys. D: Appl. Phys., 49 (2016) 295206-295216.

[7] J. Menser, K. Daun, T. Dreier, C. Schulz, Laser-induced incandescence from laser-heated silicon nanoparticles, Appl. Phys. B, 122 (2016) 277-293.

[8] J. M. Mitrani, M. N. Shneider, Time-resolved laser-induced incandescence from multiwalled carbon nanotubes in air, Appl. Phys. Lett., 106 (2015) 043102-043106.

[9] M. N. Shneider, Carbon nanoparticles in the radiation field of the stationary arc discharge, Phys. Plasmas, 22 (2015) 073303-073307.

[10] J. M. Mitrani, M. N. Shneider, B. C. Stratton, Y. Raitses, Modeling thermionic emission from laser-heated nanoparticles, Applied Physics Letters, 108 (2016) 054101-054105.

[11] C. Uher, Thermal Conductivity of Pure Metals and Alloys, Volume 15c of the series Landolt-Börnstein - Group III Condensed Matter , (1991) 430-439. 
[12] B. Angleraud, F. Garrelie, F. Tetard, A. Catherinot, Study of particles ejected after pulsed laser ablation of a graphite target, Appl. Surface Sci., 138-139 (1999) 507-511.

[13] S. Gershman and Y. Raitses, Unstable behavior of anodic arc discharge for synthesis of nanomaterials, J. Phys. D Appl. Phys, 49(2016) 345201-3452010.

[14] V. Vekselman, B. Stratton, Y. Raitses, The layered structure of the carbon arc discharge plasma, APS Division of Plasma Physics Meeting, Nov. 2016, San-Jose CA US.

[15] V. A. Nemchinsky and Y. Raitses, Atmospheric pressure arc discharge with ablating graphite anode, J. Phys. D Appl. Phys., 48 (2015) 245202-245210.

[16] K. J. Daun, G. J. Smallwood, F. Liu, Investigation of Thermal Accommodation Coefficients in Time-Resolved Laser-Induced Incandescence, Journal of Heat Transfer, 130 (2008) 121201121210.

[17] F. Liu, K. J. Daun, D.R. Snelling, G. J. Smallwood, Heat conduction from a spherical nanoparticle: Status of modeling heat conduction in laser-induced incandescence, Applied Physics B: Lasers and Optics, 83 (2006) 355-382.

[18] B. J. McCoy, C.Y. Cha, Transport phenomena in the rarefied gas transition regime, Chemical Engineering Science, 29 (1974) 381-388.

[19] D. J. Collins, R. Greif, A.E. J. Bryson, Measurement of the thermal conductivity of helium in the temperature range $1600-6700{ }^{\circ} \mathrm{K}$, Int. J. Heat Mass Transfer, 8 (1965) 1209-1216.

[20] H. A. Michelsen, F. Liu, B. F. Kock, H. Bladh, A. Boiarciuc, M. Charwath, T. Dreier, R. Hadef, M. Hofmann, J. Reimann, S. Will, P. E. Bengtsson, H. Bockhorn, F. Foucher, K. P. Geigle, C. Mounaïm-Rousselle, C. Schulz, R. Stirn, B. Tribalet, R. Suntz, Modeling laserinduced incandescence of soot: A summary and comparison of LII models, Applied Physics B: Lasers and Optics, 87 (2007) 503-521.

[21] C.F. Bohren, D. R. Huffman, Absorption and scattering of light by small particles, New York: Wiley, 1983. 
[22] J. Janzen, The refractive index of colloidal carbon, Journal of Colloid and Interface Science, 69 (1979) 436-447.

[23] C. Mätzler, MATLAB functions for Mie scattering and absorption, Institut für Angewandte Physik Research Report No. 2002-08, University of Bern, 2002. 


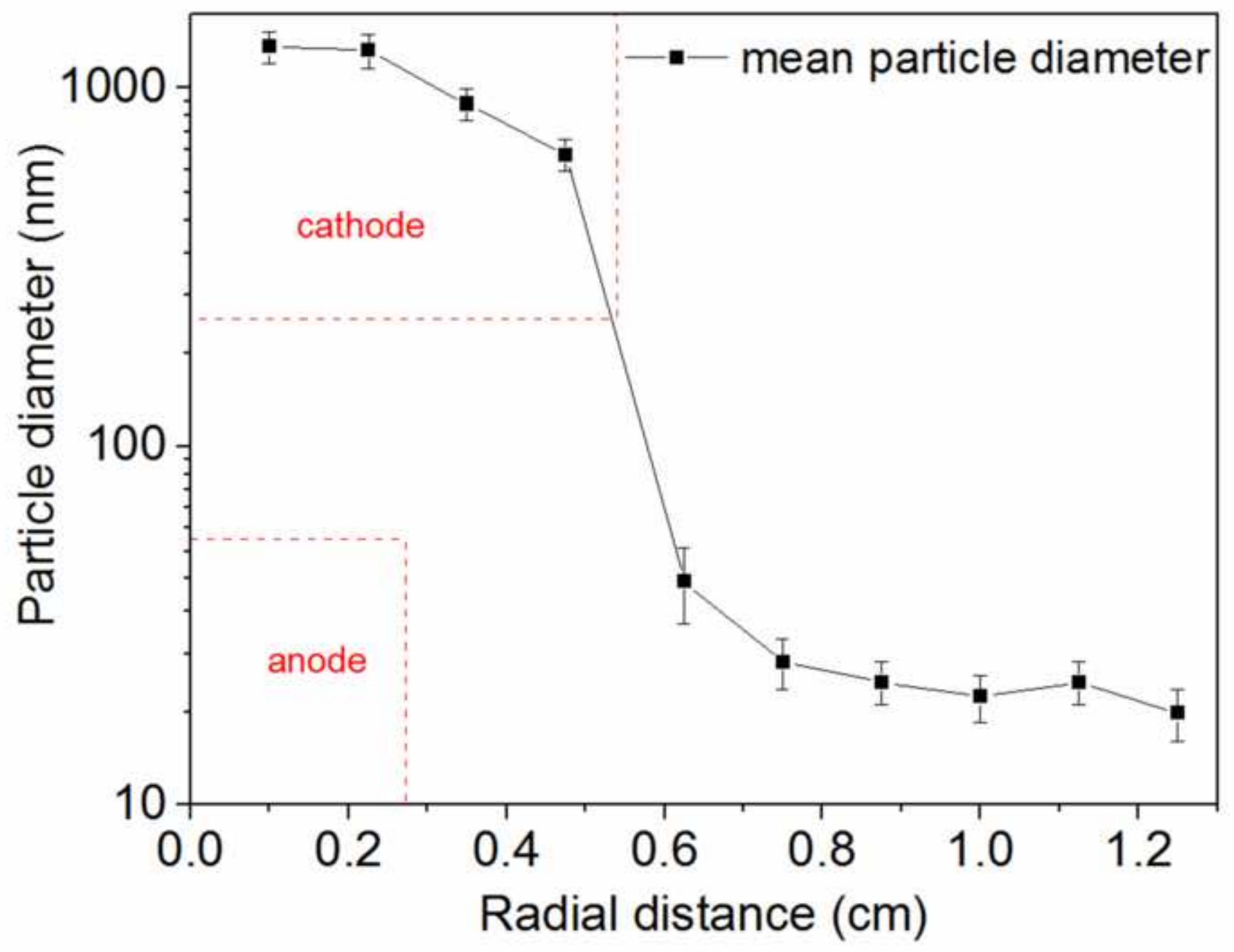

